\title{
Visualization on Formation Process of Wrinkling Phenomena on Thin Membranes Using Grating Projection Method and Investigation on Their Nonlinear Mechanical Properties
}

\author{
By Hiroshi KaWAmUrA ${ }^{1)}$, Takashi Iwasa ${ }^{1)}$, Keita Unemitsu ${ }^{2)}$, Ryo SugiYama ${ }^{3)}$ \\ Naoko KISHIMOTO ${ }^{4)}$, Ken HigUCHI ${ }^{5)}$, Motoharu FUJIGAKI ${ }^{6}$ and Takayuki SHIOKAWA ${ }^{6}$ \\ ${ }^{1)}$ Department of Mechanical and Aerospace Engineering, Tottori University, Tottori, Japan \\ ${ }^{2)}$ Hiroshima Aluminum Industry Co., Ltd., Hiroshima, Japan \\ ${ }^{3)}$ Imasen Electric Industrial Co., Ltd., Inuyama, Japan \\ ${ }^{4)}$ Department of Mechanical Engineering, Setsunan University, Osaka, Japan \\ ${ }^{5)}$ Division of Aerospace Engineering, Muroran Institute of Technology, Muroran, Japan \\ ${ }^{6}$ Department of Opto-Mechatronics, Wakayama University, Wakayama, Japan
}

(Received June 27th, 2011)

\begin{abstract}
A non contact whole field shape measurement using the grating projection method was performed to visualize formation and dissipation processes of the wrinkling phenomena on thin membranes, and their nonlinear mechanical properties were discussed. The wrinkling phenomena in a rectangular polyimide film, which held fixed at the bottom side and subjected to loadings at the upper side, was treated. The measurement results showed that the nonlinear wrinkling behavior such as the snap through phenomena appeared in the formation and dissipation processes of wrinkles was successfully captured. Through the discussion on the dissipation processes of wrinkling phenomena, the conditions of the applied tensions to reduce the distortion of the membrane surface feature are presented.
\end{abstract}

Key Words: Whole Field Shape Measurement, Formation and Dissipation Process of Wrinkling Phenomena, Grating Projection Method

Nomenclature

$$
\begin{array}{cll}
x & : & \text { x position coordinate } \\
y & : & \text { y position coordinate } \\
z & : & \text { z position coordinate } \\
\phi & : & \text { phase of grating patterns } \\
\text { Subscripts } & & \\
i & : & \text { reference plane, } i=1,2
\end{array}
$$

\section{Introduction}

A membrane is considered as one of the effective structural components for flexible large space structures such as solar sails, large aperture antennas and sunshields. However, since the membrane has negligible bending stiffness and can carry no compressive stresses, it is easily deformed due to small external forces. Especially, wrinkles and slacks induced by the deformation processes may affect the operational functions of the membrane space structures. Therefore, to establish the membrane structures with wrinkle free design, it is important to clarify the mechanical properties of the formation processes of the wrinkling phenomena in membranes, and to find the condition of the tensile loadings to control the distortion of the membrane surface features with wrinkles.

Currently, a great number of studies on wrinkling phenomena have been reported. In the experimental studies of an early date, the local behavior of the wrinkled membranes have been measured by the non contact displacement sensor, and effects of the tensile and shear loadings on wrinkling features have been discussed $^{1,2)}$. Jenkins et al. also investigated the condition of the tensile and shear loadings for occurrence of wrinkles based on the visual judgment of the photograph ${ }^{1)}$. However, to investigate the loading effects on the overall wrinkling behavior quantitatively, the non contact whole field shape measurements with high accuracy should be performed.

To respond the demands, the studies on the non contact whole field shape measurement for membrane structures have received wide attention during the last decades ${ }^{3-6)}$. In these studies, the photogrammetry measurements using circular targets have been focused upon, and effectiveness of these measurements method for the membrane structures has been discussed. However, in the photogrammetry measurement using the targets, the spatial resolution of the surface configuration of the wrinkled membranes is directly affected by the number and size of the targets. This implies that it is difficult to measure the detailed membrane surface feature including small wrinkles, quantitatively.

Recently, the various non contact measurement systems capable of measuring the characteristics of membrane space structures have been reviewed ${ }^{7}$. And, the photogrammetry measurements have also been applied to study the effectiveness of the active flatness control of the membranes structure ${ }^{8)}$.

In this paper, in order to investigate the nonlinear mechanical properties of the formation processes of the wrinkling phenomena in detail, the whole field shape measurement using the grating projection method was conducted. Since the grating projection method is able to measure the $3 \mathrm{D}$ position coordinates of the object by a pixel to pixel basis, the membrane surface features including small wrinkles can be measured in detail. However, this method cannot measure the strain field of the object due to its measurement characteristics. Accordingly, this work was 
performed focusing on the measurement of the surface configuration of the membranes with wrinkles and slacks subjected to in-plane loadings.

Firstly, the measurement accuracy of the grating projection method used in our laboratory was investigated. Secondly, the formation and dissipation processes of the wrinkling phenomena in thin membranes were visualized. Through the discussion on the visualized data, the relation of the wrinkle features to the applied loadings was studied. Finally, the condition of the applied tensions to reduce the wrinkle features was discussed to indicate the guidelines for wrinkle free design of the future space structures incorporating membranes.

\section{Outline of Experiment}

\subsection{Grating projection method}

The grating projection method is an image analysis method to calculate the $3 \mathrm{D}$ position coordinate of the object with high speed and high accuracy by analyzing the phase of the grating patterns projected to the object. The grating pattern is projected by the projector. The image data to analyze the phase are photographed by a digital camera. Unlike the general non contact 3D shape measurement method such an image correlation method, the grating projection method requires only one camera to obtain the 3D position coordinates of the objects. In addition, since the grating projection method provides the $3 \mathrm{D}$ position coordinates of the object by a pixel to pixel basis, the spatial resolution becomes higher than the non contact 3D shape measurement method such an image correlation method. In this work, the grating projection method, which uses the two reference planes in the calibration stages, was applied to prevent the lens aberration effects ${ }^{9}$.

Figure 1 indicates the outline of the measurement system of the grating projection method used in our work. As shown in the figure, the coordinate and phase of the arbitrary point on the object are represented by $(x, y, z, \phi)$, and those of the two reference planes that are installed on the back and forth of the object are indicated by $\left(x_{1}, y_{1}, z_{1}, \phi_{1}\right),\left(x_{2}, y_{2}, z_{2}, \phi_{2}\right)$. The $\mathrm{x}$, $\mathrm{y}$ axes were set to be the horizontal and vertical direction of the reference plane, and the $\mathrm{z}$ axis was set perpendicular to the reference plane. Here, when the coordinate and phase are assumed to be varied linearly from the reference plane to another one, the $3 \mathrm{D}$ coordinate of the object installed between the two reference planes can be calculated from the following equations.

$$
\begin{gathered}
x=\frac{\phi-\phi_{1}}{\phi_{2}-\phi_{1}}\left(x_{2}-x_{1}\right)+x_{1} \\
y=\frac{\phi-\phi_{1}}{\phi_{2}-\phi_{1}}\left(y_{2}-y_{1}\right)+y_{1} \\
z=\frac{\phi-\phi_{1}}{\phi_{2}-\phi_{1}}\left(z_{2}-z_{1}\right)+z_{1}
\end{gathered}
$$

From the equations, if the $3 \mathrm{D}$ coordinates and phases of the reference planes were preliminary obtained, the 3D coordinate of the object can be calculated from only measuring the phase of the grating patterns projected to the object.

In the calculation on the phase, two typical methods have been proposed, namely sampling moiré method ${ }^{10)}$ and phase-shifting method $^{11)}$. Of these two methods, the sampling moiré method is characterized by the high computational efficiency, while the resolution of this method is low compared to the phase-shifting method. In this work, the sampling moiré method was applied for the measurement. This is because this method is suitable for sensing the dynamic responses of the object and is considered to be a scalable method in our future work.

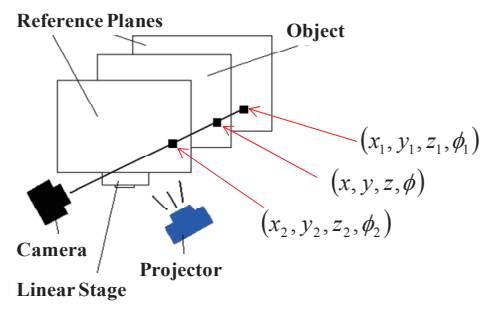

Fig. 1. Outline of measurement system.

\subsection{Test equipment}

Figure 2 indicates the picture of the rectangular polyimide film and the test equipment. As shown in Fig. 2(a), two acrylic boards were attached at a distance of $0.16 \mathrm{~m}$ on the upper and bottom edges of the rectangular polyimide film. The two acrylic boards were attached by a dedicated instrument so that the acrylic boards were precisely placed side by side. The width of the rectangular film was set to be $0.30 \mathrm{~m}$, and the thickness was $12.5 \times 10^{-6} \mathrm{~m}$. In this work, the film surface was preliminary matted to prevent the gloss effects for the measurement. Table 1 lists the parameter of the polyimide film used in the work.

When the polyimide film was mounted on the test equipment, these acrylic boards were clamped by the clamp bars mounted on the test equipment as shown in Fig. 2(b). The distance of the upper and bottom clamp bars of the test equipment were set to be $0.16 \mathrm{~m}$ so that the polyimide film has even surface and no stress field. However, as shown in Fig. 2(b), since it is difficult to set up the distance of these clamp bars strictly, the slack regions appeared in the rectangular polyimide film at the installation stages.

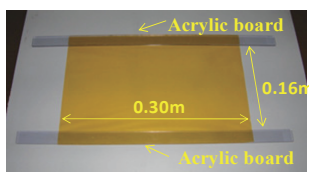

(a) Rectangular polyimide film

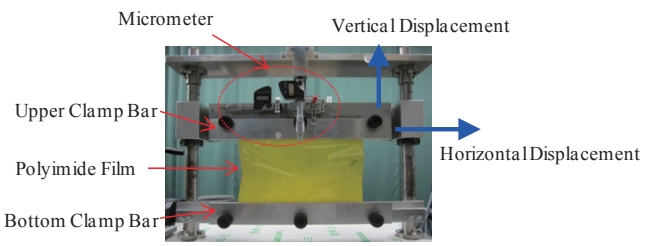

(b) Test equipment.

Fig. 2. Polyimide film and test equipment.

On the upper part of the test equipment, two micrometers were attached so that vertical and horizontal displacements can be enforced to the upper clamp bar. Due to these enforced 
displacements, the tensile and shear loadings were applied to the rectangular polyimide film, and the wrinkling phenomena appeared. In this work, these wrinkling phenomena were measured by the grating projection method.

Table 1. Parameter of polyimide film.

\begin{tabular}{|l|c|}
\hline Polyimide Film & Kapton H-Type \\
\hline Young's Modulus & $3500 \mathrm{MPa}$ \\
\hline Poisson's Ratio & 0.3 \\
\hline Size of the Film & $0.30 \mathrm{~m} \times 0.16 \mathrm{~m}$ \\
\hline
\end{tabular}

\subsection{Measurement system}

Figure 3 indicates the picture and the detailed layout of the measurement system. A digital camera and a projector were attached to the measurement jig, and they were placed $0.30 \mathrm{~m}$ away from each other. This measurement jig was installed on a single-axis spindle stage, which can be moved back and forth with positioning accuracy $\pm 15 \times 10^{-6} \mathrm{~m}$. The measurement jig was installed in front of the test equipment at a distance of $1.0 \mathrm{~m}$. Outline of the measurement equipment was listed in Table. 2.

Table 2. Outline of measurement equipment.

\begin{tabular}{|l|l|}
\hline Digital Camera & $\begin{array}{l}\text { Dragonfly Express Point Gray } \\
\text { Effective Pixels: 640x680 }\end{array}$ \\
\hline Projector & $\begin{array}{l}\text { EPSON, EB-1730W } \\
\text { Effective Pixels: } 1280 \times 800\end{array}$ \\
\hline Single-Axis Spindle Stage & $\begin{array}{l}\text { SIGUMA KOKI, SG SP26-200 } \\
\text { Positioning Accuracy: } \pm 15 \times 10^{-6} \mathrm{~m}\end{array}$ \\
\hline
\end{tabular}

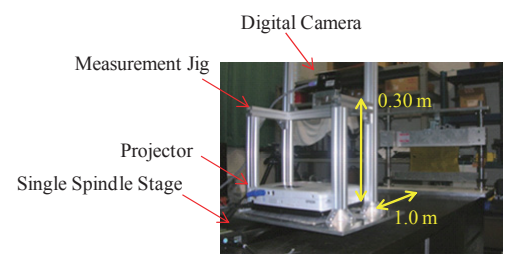

(a) Picture of measurement system

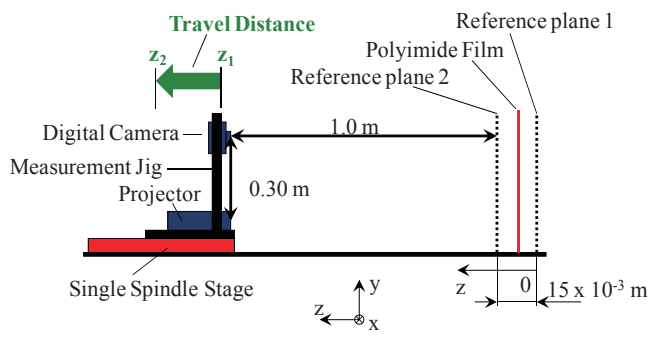

(b) Layout of measurement system

Fig. 3. Measurement system.

\subsection{Preliminary experiment}

In order to evaluate the measurement accuracy of the grating projection method used in this work, the preliminary measurement was performed.

Figure 4 shows the measurement situation of the mat polyimide film. In this experiment, a tensile loading was firstly applied by enforcing the vertical displacement to the upper clamp bar until the film was taut. Then, 3D position coordinate of the membrane surface was measured by the grating projection method. This measurement was performed four times with changing the distance between the membrane and the measurement jig. This distance was adjusted by the movement of the measurement jig, which was controlled by the single-axis spindle stage. The travel distance of the measurement jig due to the single-axis spindle stage, which was shown in Fig. 3(b), is listed in Table 3. This value has an uncertainty relating to the positioning accuracy $\pm 15 \times 10^{-6} \mathrm{~m}$ of the single axis spindle stage.

The measurement accuracy of the grating projection method was discussed in the following section by comparing the travel distance of the measurement jig to that calculated from the $3 \mathrm{D}$ position coordinates of the membranes measured by the grating projection method.

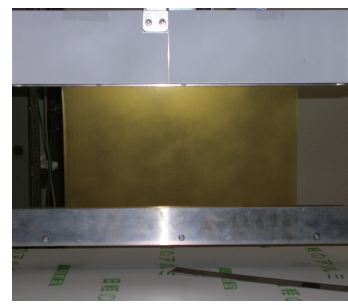

Fig. 4. Measurement situation of mat polyimide film.

Table 3. Travel distance of measurement jig.

\begin{tabular}{|c|c|c|c|c|}
\hline Number of Movement & 1 & 2 & 3 & 4 \\
\hline Travel Distance $\left[\mathrm{x} 10^{-3} \mathrm{~m}\right]$ & 3 & 6 & 9 & 12 \\
\hline
\end{tabular}

\subsection{Measurement accuracy}

Figure 5 indicates the measurement results of the mat polyimide film in the initial measurement (Number of Movement: 1 in Table 3). Figure 5(a) indicates the $\mathrm{z}$ position coordinate of the film by the contours, and Fig. 5(b) indicates the cross sectional shape of the membranes, which was shown in the dotted line in Fig. 5(a). From the figures, measurement results on the $\mathrm{z}$ position coordinate were different by location and varied on each pixel, although the film was tauten. It is considered that these results occurred due to the gross effects of the membrane surface, which cannot be perfectly removed by the matting.

Table 4 lists the measurement results. In this table, "Travel Distance" indicates the movement of the measurement jig due to the single spindle stage, which was listed in Table 3. "Measured Average Distance" represents the distance calculated from the $\mathrm{z}$ position coordinates of the membranes measured by the grating projection method. In the grating projection method, since the $\mathrm{z}$ position coordinate was measured on a pixel to pixel basis and varied by location as stated above, the average and standard deviation of the measured $\mathrm{z}$ position coordinates were calculated by using the data within the rectangular regions as shown in Fig. 5. The rectangular region used in the calculation was selected so that the measurement results indicating the shape of the micrometers, upper and bottom clamp bars were excluded. The number of pixels in the rectangular regions was 140021. Accordingly, "Measured Average Distance" was derived from the calculated average values on the $\mathrm{z}$ position coordinates of the membranes. "Standard Deviation" in Tab. 4 is the calculation results of the measured $\mathrm{z}$ position coordinate stated above, and indicates the measurement variation. "Measurement Errors" listed in Tab. 4 indicates the differences between the values in "Travel Distance" and "Measured Average Distance". 
From the results on "Measurement Errors", it turned out that the $\mathrm{z}$ position coordinates of the mat polyimide film can be measured by the grating projection method with about $0.130 \times 10^{-3}$ $\mathrm{m}$ accuracy. Incidentally, the $95 \%$ confidence interval for "Measured Average Distance" can be calculated from the following equation.

$$
\delta_{95}=\frac{t_{N-1}(0.95) \cdot \sigma}{\sqrt{N}}
$$

Here, $t_{N-1}(0.95), \sigma, N$ in Eq. (4) indicate t-value with $95 \%$, standard deviation and number of the data, respectively. The $95 \%$ confidence interval for "Measured Average Distance" calculated from Eq. (4) was about $0.69 \times 10^{-6} \mathrm{~m}$. These results imply that the shape measurement with the grating projection method used in this work is suitable for measuring the amplitude of the wrinkles larger than $1.30 \times 10^{-3} \mathrm{~m}$ at least.

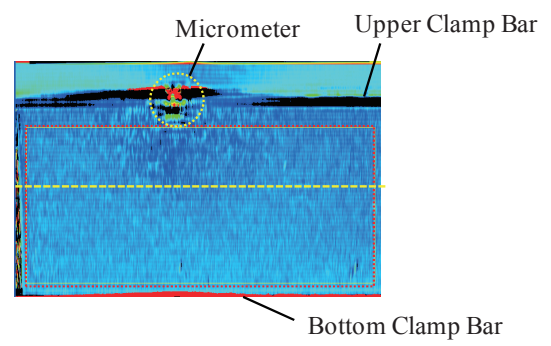

(a) Whole field shape measurement results

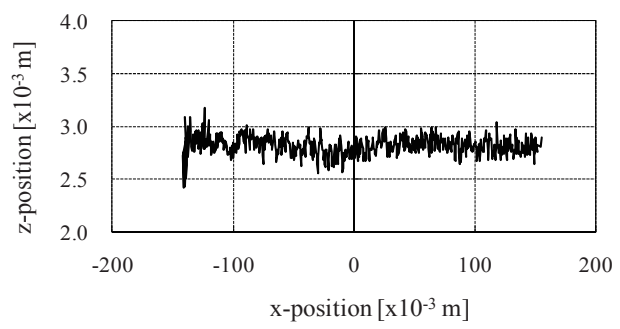

(b) Cross sectional deformation

Fig. 5. Measurement results on polyimide film at initial measurement.

Table 4. Measurement results.

\begin{tabular}{|c|c|c|c|c|}
\hline Number of Movement & 1 & 2 & 3 & 4 \\
\hline Travel Distance & 3.000 & 6.000 & 9.000 & 12.000 \\
\hline Measured Average Distance & 2.868 & 5.869 & 8.893 & 11.910 \\
\hline Standard Deviation & 0.132 & 0.134 & 0.133 & 0.129 \\
\hline Measurement Errors & 0.132 & 0.131 & 0.107 & 0.090 \\
\hline
\end{tabular}

Unit: $\left[\times 10^{-3} \mathrm{~m}\right]$

\section{Results and Discussion}

\subsection{Experiment cases}

The nonlinear mechanical properties of the wrinkling phenomena and the condition of the applied tensions to control the wrinkling phenomena in the membranes were discussed. Table 5 indicates the experiment cases performed in this work.

In Cases 1 and 2, the enforced horizontal and upward vertical displacements, which were applied to the upper clamp bar, were set to be the same, while the loading sequences were reverse. These two cases were conducted to investigate the effects of the loading sequences on the formation and dissipation processes of the wrinkling phenomena in the membranes. In Cases 3 and 4, the loading sequences were set to be the same, while the enforced displacements were different. These two cases were carried out to study the condition of the tensile loading to remove the wrinkling phenomena in the membranes.

In all cases, the enforced horizontal and upward vertical displacements were applied by $0.1 \times 10^{-3} \mathrm{~m}$ step, and the surface configurations of the membranes were measured at each step.

Table 5. Experiment cases.

\begin{tabular}{|c|l|l|}
\hline Cases & \multicolumn{1}{|c|}{ Enforced Displacement } & Loading Sequences \\
\hline Case 1 & $\begin{array}{l}\text { Horizontal }: \mathrm{dh}=0.5 \times 10^{-3} \mathrm{~m} \\
\text { Vertical: } \quad \mathrm{dv}=0.5 \times 10^{-3} \mathrm{~m}\end{array}$ & Horizontal $\rightarrow$ Vertical \\
\hline Case 2 & $\begin{array}{l}\text { Horizontal }: \mathrm{dh}=0.5 \times 10^{-3} \mathrm{~m} \\
\text { Vertical: } \quad \mathrm{dv}=0.5 \times 10^{-3} \mathrm{~m}\end{array}$ & Vertical $\rightarrow$ Horizontal \\
\hline Case 3 & $\begin{array}{l}\text { Horizontal }: \mathrm{dh}=0.3 \times 10^{-3} \mathrm{~m} \\
\text { Vertical: } \quad \mathrm{dv}=0.7 \times 10^{-3} \mathrm{~m}\end{array}$ & Horizontal $\rightarrow$ Vertical \\
\hline Case 4 & $\begin{array}{l}\text { Horizontal }: \mathrm{dh}=0.5 \times 10^{-3} \mathrm{~m} \\
\text { Vertical: } \quad \mathrm{dv}=1.0 \times 10^{-3} \mathrm{~m}\end{array}$ & Horizontal $\rightarrow$ Vertical \\
\hline
\end{tabular}

\subsection{Formation and dissipation processes of wrinkles}

Figures 6 and 7 show the shape measurement results on wrinkled membranes in Cases 1 and 2, respectively. These results indicate the formation and dissipation processes of the wrinkles and slacks in the membrane subjected to different loading sequences. The surface configuration of the wrinkled membranes at each step was successfully measured except the dotted circle area. It is considered that the disturbances observed in these areas were caused by the gross effects of the membrane surface and due to the phase calculation method based on the sampling moiré method used in this work.

Figure 8 indicates the comparison on the formation and dissipation processes of the wrinkles and slacks focusing on the cross-sectional deformations in Cases 1 and 2. This comparison was performed in order to investigate the effects of the loadings sequences on the final surface configuration of the wrinkled membranes from the view points of the formation and dissipation processes of the wrinkles and slacks. In Case1, when the horizontal displacement was applied, slacks in the right side region observed in the initial state were firstly moving forward (Steps2-3), and single wrinkle occurs in the middle regions (Step 4). Then, several wrinkles formed as the horizontal displacement was increased (Steps5-6). After that, as the enforced vertical displacement increases (Steps7-11), the amplitude of wrinkles becomes small with the occurrence of the snap through phenomena. Due to the phenomena, the overall wrinkle features change (Steps7-8) and novel wrinkles appear around the previous wrinkles (dotted circle areas in Steps8-11). On the other hand, in Case 2, when the vertical displacement applied to the membranes increases (Steps1-6), the slacks in the both right and left side regions in the initial state only diminishes, and wrinkles did not occur. After that, by applying the enforced horizontal displacement (Steps7-11), several wrinkles appeared.

Comparing the results of Case 1 with Case 2, it was clear that the formation processes of wrinkles were significantly affected by the loading sequences. However, focusing on the final state of the 
wrinkled membrane features (Step11), the results of the two cases were in good agreement. And this agreement occurred when the final enforced displacement was applied (Steps10-11). These results indicate that the final wrinkled membrane features were not affected by the differences of the loading sequences, while the formation processes were affected. In our previous studies, however, it is pointed out that the dependency of the final membrane surface features on the loading sequences may appear ${ }^{12)}$, and the result stated above does not always happen. It is considered that these differences occur due to the effects of the initial condition of the membranes such as initial shape and initial stress fields.

Figure 8 also shows that the wrinkling phenomena in the membranes occur due to shear loadings, and due to tensile loadings, the amplitude of the wrinkles becomes small. This implies that the tensile loadings are one of the simplified factors to control the wrinkled membrane features. Accordingly, the condition of the upward vertical displacement to reduce the wrinkling phenomena was discussed in the following section.

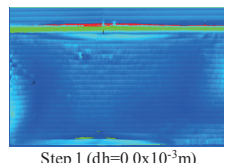

Step $1\left(\mathrm{dh}=0.0 \times 10^{-3} \mathrm{~m}\right)$

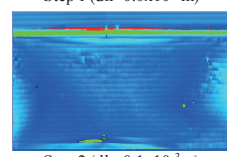

Step $2\left(\mathrm{dh}=0.1 \times 10^{-3} \mathrm{~m}\right)$

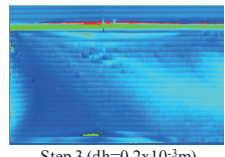

$\operatorname{Step} 3\left(\mathrm{dh}=0.2 \times 10^{-3} \mathrm{~m}\right)$

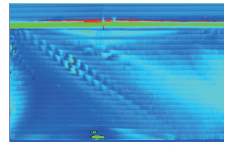

Step $4\left(\mathrm{dh}=0.3 \times 10^{-3} \mathrm{~m}\right)$

Fig. 6. Whole field shape measurement results in Case 1.

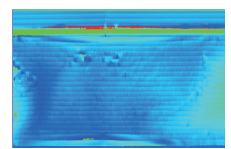

Step $1\left(\mathrm{dv}=0.0 \times 10^{-3} \mathrm{~m}\right)$

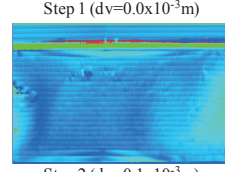

$\operatorname{Step} 2\left(\mathrm{dv}=0.1 \times 10^{-3} \mathrm{~m}\right)$

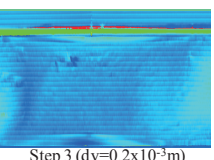

Step $3\left(\mathrm{dv}=0.2 \times 10^{-3} \mathrm{~m}\right)$

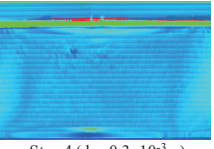

$\operatorname{tep} 4\left(\mathrm{dv}=0.3 \times 10^{-3} \mathrm{~m}\right)$

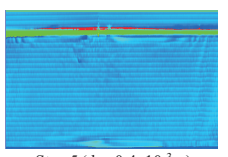

$\operatorname{Sten} 5\left(\mathrm{dv}=0.4 \times 10^{-3} \mathrm{~m}\right)$
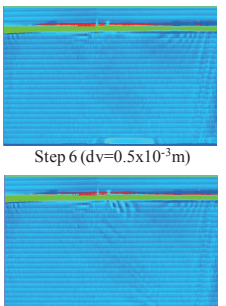

tep $7\left(\mathrm{dh}=0.1 \times 10^{-3} \mathrm{~m}\right)$

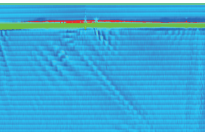

Step $8\left(\mathrm{dh}=0.2 \times 10^{-3} \mathrm{~m}\right)$

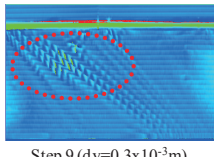

Step $9\left(\mathrm{dv}=0.3 \times 10^{-3} \mathrm{~m}\right)$

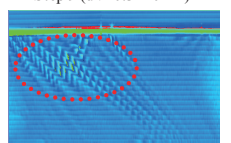

Step $10\left(\mathrm{dv}=0.4 \times 10^{-3} \mathrm{~m}\right)$

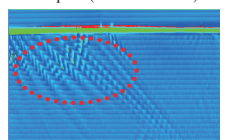

Step $11\left(\mathrm{dv}=0.5 \times 10^{-3} \mathrm{~m}\right)$
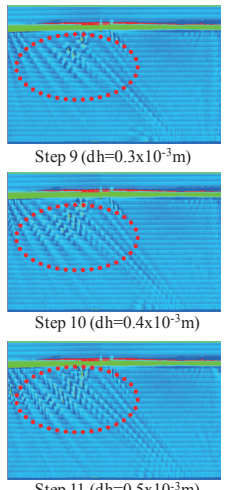

Step $11\left(\mathrm{dh}=0.5 \times 10^{-3} \mathrm{~m}\right)$
Fig. 7. Whole field shape measurement results in Case 2.
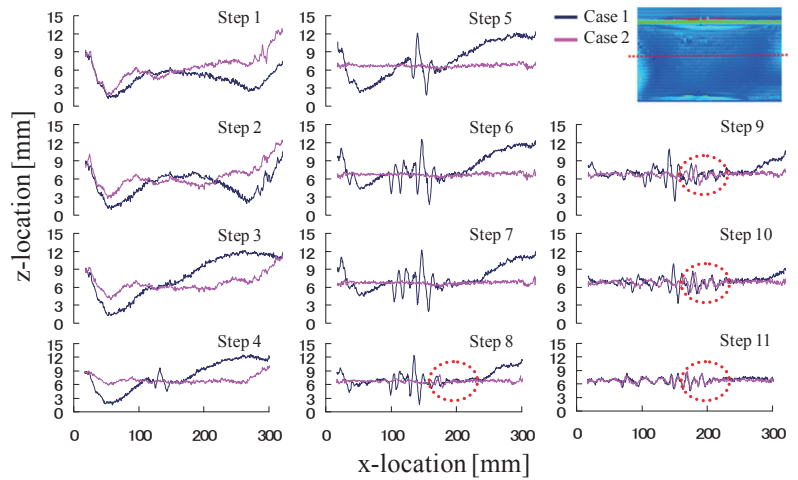

Fig. 8. Formation and dissipation process of wrinkling phenomena.

\subsection{Tension condition to control wrinkles}

Focusing on the dissipation processes of the wrinkling phenomena in the membranes, the condition of the applied tension to reduce the wrinkling phenomena was investigated.

Figures 9 and 10 indicate the measurement results of wrinkled membranes in Cases 3 and 4, respectively. These figures show the dissipation processes of the wrinkling phenomena in the membranes when the enforced vertical displacement was applied. Namely, the results in Step 1 in these figures correspond to the surface configurations of the wrinkled membranes after the enforced horizontal displacement was fully applied. From the figure, the amplitude of the wrinkles becomes small, and finally diminishes due to the upward vertical displacement.

To investigate the dissipation processes of the wrinkling phenomena quantitatively, the RMS value of the out-of-plane deformation of the membrane surface was calculated for representing the distortion of the wrinkled membrane surface. And its variation in the dissipation processes of the wrinkling phenomena is discussed. Since the grating projection method provides the $3 \mathrm{D}$ position coordinate by a pixel to pixel basis, the RMS value can be obtained directly from the measured pixel data. The wrinkled region used in the calculation is shown in Fig. 11. This region is selected so that the slack regions were excluded. The size of the region is $0.16 \mathrm{~m} \times 0.15 \mathrm{~m}$, and the number of the pixels in the rectangular region was 108160 .
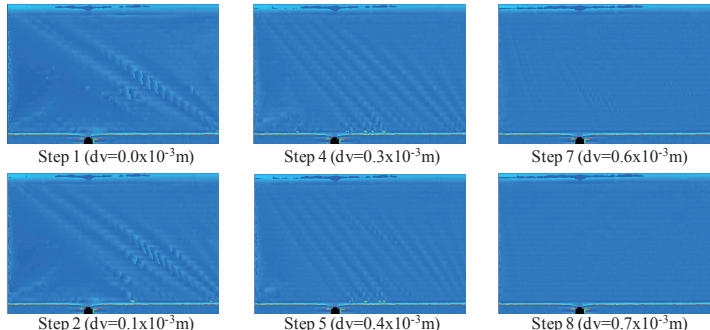

Step $7\left(\mathrm{dv}=0.6 \times 10^{-3} \mathrm{~m}\right)$
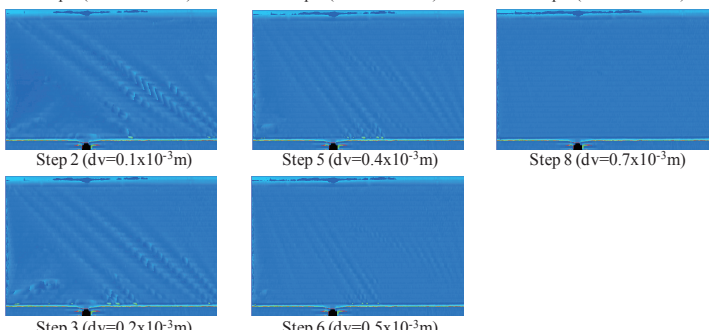

Step $8\left(\mathrm{dv}=0.7 \times 10^{-3} \mathrm{~m}\right)$

Fig. 9. Whole field shape measurement results in Case 3. 


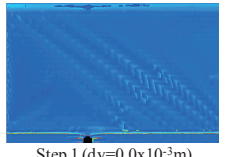

Step $1\left(\mathrm{dv}=0.0 \times 10^{-3} \mathrm{~m}\right)$

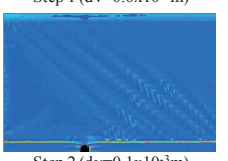

Step $2\left(\mathrm{~d} v=0.1 \times 10^{-3} \mathrm{~m}\right)$
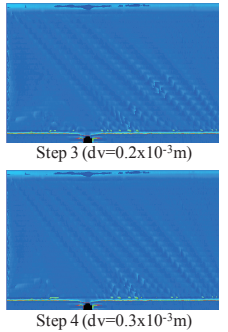

Fig. 10. Whole field shape measurement results in Case 4.

Figure 12 indicate the relationship between the distortion of the membrane surface features and the applied vertical displacement. From the figure, it turns out that the distortion of the membrane surface features in both cases monotonically decreases, and approaches asymptotically to the final value. This final value was about $0.30 \times 10^{-3} \mathrm{~m}$, and does not vanish perfectly even considering the measurement variation (roughly $0.130 \times 10^{-3} \mathrm{~m}$ ) listed in Table 5. This result indicates that the distortion of the membrane surface features cannot be removed perfectly with the tension loadings.

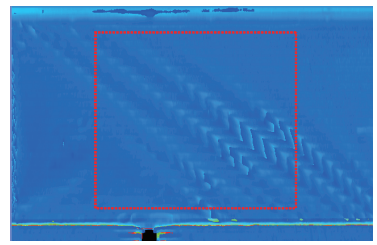

Fig. 11. Selected wrinkled regions.

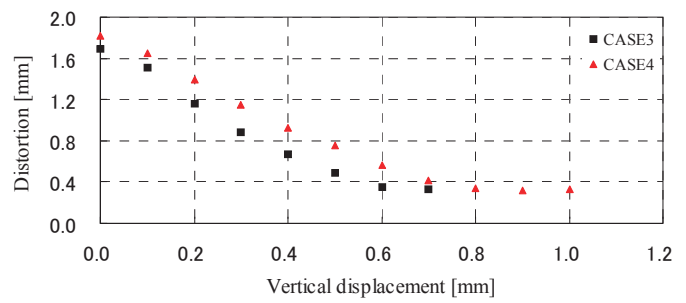

Fig. 12. Reduction processes of membrane feature distortions.

Figure 13 indicate the normalized expression of Fig. 12. In the abscissa, the enforced vertical displacement was normalized by the final enforced horizontal displacement applied to the membranes. This normalized displacement used in the abscissa physically represents the conclusive geometrical position relation of the boundary movement regarding the vertical and horizontal direction. If a linear strain relation was assumed, the normalized displacement was equivalent to the ratio of the normal strain to shear strain.
In the ordinate, the distortion of the membrane surface features is normalized by its initial value, namely reduction rate. This figure shows that the final asymptotic values in both cases became roughly 0.2 . Therefore, the controllable range of the distortion of the membrane surface features with the tension loadings is about $80 \%$ reduction from the initial states in the treated models here.

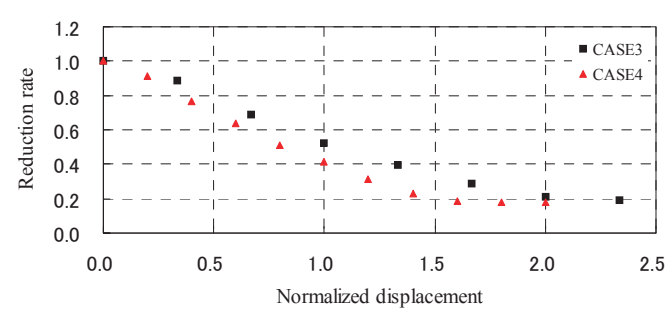

Fig. 13. Reduction rate of the distortion.

By using the results shown in this figure, the geometrical relation of the boundary movement, which is necessary to control the distortion of the membrane surface features, can be simply estimated. For example, when the $50 \%$ reduction of the distortion from the current states is considered, the same movement as the horizontal displacement currently applied is necessary. It is considered that these relations are useful for the estimation of the tension loadings to keep mirror accuracy of the membranes within the predetermined value. For example, when the horizontal displacement in the boundary is preliminary obtained as a clearance, backlash and play in the design, the necessary tension loadings can be easily estimated from the figure.

However, reliable reduction curves such as a regression curve have not been obtained yet, since the number of the experiment cases is not sufficient at the present time. To provide more useful data, much more experiments are necessary, and the statistical investigations on these reduction curves have to be conducted.

\section{Conclusions}

A non contact whole field shape measurement with the grating projection method was applied to visualize formation and dissipation processes of the wrinkling phenomena on thin membranes, and the nonlinear mechanical properties of wrinkling phenomena were discussed. The conclusions obtained in this work were summarized below.

(1) The measurement accuracy of the grating projection method with the sampling moire method used in this work is about $0.130 \times 10^{-3} \mathrm{~m}$ for the measurement of a rectangular mat polyimide film of $0.30 \mathrm{~m} \times 0.16 \mathrm{~m}$.

(2) The formation and dissipation processes of wrinkling phenomena in membranes were quantitatively visualized, and the nonlinear membrane behavior including snap through phenomena of wrinkles was successfully captured.

(3) The distortion of the membrane surface features cannot be removed perfectly with the tension loadings, and the 
controllable range of the distortion with the tension loadings becomes about $80 \%$ reduction from the initial state in the cases treated here.

(4) The geometrical relation regarding to the boundary movement, which was necessary to control the distortion of the membrane surface feature, was experimentally presented. For example, in the model treated here, if the $50 \%$ reduction of the distortion from the current states is required, the same vertical movement as the horizontal displacement currently applied is necessary.

\section{Acknowledgments}

This work was supported by the Grant-in-Aid (B), No.21560824, the ministry of education, culture, sports, science and technology.

\section{References}

1) Jenkins, C. H., Haugen, F. and Spicher W.H.: Experimental Measurement of Wrinkling in Membranes Undergoing Planar Deformation, Experimental Mechanics., 38, No. 2 (1998), pp.147-151.

2) Reynolds, R. R., Ferguson T. P. and Funkhouser J. C.: Measurement of Residual Wrinkles in Polymer Membranes, 42nd AIAA/ASME/ASCE/AHS/ASC Structures Structural Dynamics, and Materials Conference and Exhibit, A01-25123 (2001).

3) Giersch, L. R. M..: Pathfinder Photogrammetry Research for Ultra-Lightweight and Inflatable Space Structures, NASA/CR2001-211244, (2001).

4) Pappa, R. S., Black, J. T., Blandino, J. R., Jones, T. W., Danehy, P.M. and Dorrington, A. A.: Dot-Projection Photogrammetry and Videogrammetry of Gossamer Space Structures, NASA/TM-2003-212146, (2003).

5) Black, J. T. and Pappa, R. S.: Videogrammetry Using Projected Circular Targets: Proof-of-Concept Test, NASA/TM-2003-212148, (2003).

6) Wang, C., Du, X. and Wan, Z.: An Experimental Study on Wrinkling Behaviors and Characteristics of Gossamer Space Structures, Strains, 43 (2007), pp.332-339.

7) Roose, S., Stockman, Y., Rochus, P. Kuhn, T., Lang, M., Baier, H. Langlois, S. and Casarosa, G.: Optical methods for non-contact measurements of membranes, Acta Astonautica. 65 (2009), pp.1317-1329.

8) Orszulik, R., Shan, J. and Stachowsky, M.: Membrane structure active flatness control using genetic algorithm with online objective reweighting, Acta Astonautica, Article in Press, Corrected Proof. Elsevier (2010), doi:DOI:10.1016/j.actaastro.2010.11.009.

9) Fujigaki, M. and Morimoto, Y.: Improvement of Accuracy of Shape Measurement Using Multiple Reference Planes, Mechanical Engineering Congress, III (2000), pp.187-188. (in Japanese)

10) Ri, S., Fujigaki, M. and Morimoto, Y.: Sampling Moiré Method for Accurate Small Deformation Distribution Measurement, Experimental Mechanics, 50 (2010), pp.501-508.

11) Huimin X., Haixia S., Fulong D. Biao L. and Yongming X.: Phase shifting SEM moiré method, Optics \& Laser Technology, 36 (2004), pp.291-297.

12) Unemitsu, K., Iwasa, T., Kawamura, H., Higuchi, K., Kishimoto, N., Fujigaki, M. and Shiokawa, T.: Experimental Verification on Loading Dependence of Surface Configuration of Wrinkled Membranes by using Grating Projection Method, 26th ISAS Space Structures and Material Symposium, (2010), pp.501-508. (in Japanese) 